\title{
Pre-existing long-term memory facilitates the formation of visual short-term memory
}

Weizhen Xie ${ }^{1}$ (weizhen.xie@nih.gov, OCRID: 0000-0003-4655-6496)

Weiwei Zhang² (wewei.zhang@ucr.edu, OCRID: 0000-0002-0431-5355)

1. National Institute of Neurological Disorders and Stroke, National Institutes of Health

2. Department of Psychology, University of California, Riverside

\begin{abstract}
To understand more naturalistic visual cognition, a growing body of research has investigated how pre-existing long-term memory (LTM) influences representations and processes actively engaged in visual short-term memory (VSTM). Prior research on LTM and the representation aspect of VSTM, such as the number of retained VSTM items or VSTM storage capacity, has yielded mixed findings. However, converging evidence from different experimental approaches suggests that pre-existing LTM reliably modulates the initial process of transferring fragile sensory inputs into durable VSTM contents. Here, we review recent findings along this line of research and discuss potential mechanisms for future investigations. We find that pre-existing facilitates the formation of VSTM even though it may not replace the limited VSTM storage capacity. These findings, therefore, highlight the importance of assessing how pre-existing LTM affects the mental processes underlying naturalistic visual cognition.
\end{abstract}

\section{Introduction}

At any moment, our ability to retain the information presented in the visual world relies on our VSTM. This core mental faculty enables us to stitch different pieces of visual information across eye movements to form a coherent representation of the visual world (Hollingworth, Richard, \& Luck, 2008). It also supports higher cognition, such as fluid intelligence (e.g., Fukuda, Vogel, Mayr, \& Awh, 2010). Compromised VSTM is often seen in aging, schizophrenia spectrum disorders, and depression (e.g., Norton et al., 2020; Xie et al., 2019; Xie, Cappiello, et al., 2018; Xie, Li, et al., 2018). The functional integrity of VSTM has therefore been proposed as a transdiagnostic indicator of overall mental health (Geraldo et al., 2019).

Previous research has also referred to this core mental faculty as visual working memory, which highlights both the temporary storage of and the operation (or "working") upon visual information over a brief retention interval (Baddeley, 2012; Cowan, 2008). However, because information is rarely retained but never used in some form, the distinction between VSTM and visual working memory is subtle in most cases (Cowan, 2008; Luck, 2008). As the current chapter primarily focuses on temporary information retention, we use the term VSTM throughout the rest of the chapter. We believe the principles and observations discussed here also apply to the storage component of visual working memory. 
Conventionally, VSTM is considered a separate entity from prior knowledge stored in LTM (Atkinson \& Shiffrin, 1968). Recent theories consider VSTM as visual memory contents temporarily retained under the focus of attention (Cowan, 2001; Jonides et al., 2008; Oberauer, 2002, 2009; but see Norris, 2017). To isolate "pure" VSTM from LTM, studies of VSTM usually employ simple stimulus features such as color, orientation, and novel shape that are void of rich prior LTM information (Cowan, 2001; Luck, 2008). This practice, however, compromises on rich details from our everyday visual environment (Brady et al., 2019; Orhan \& Jacobs, 2014). Emerging research in visual memory has therefore used more complex stimuli (e.g., Brady et al., 2019; Buttle \& Raymond, 2003; Curby, Glazek, \& Gauthier, 2009; Sørensen \& Kyllingsbæk, 2012; Xie \& Zhang, 2017b). These efforts improve our understanding of the organization of human memory and facilitate the application of cognitive training to potentially improve VSTM and its associated higher cognitive functions (Schwarb, Nail, \& Schumacher, 2016; Shipstead, Redick, \& Engle, 2012; but see Chen, Yee Eng, \& Jiang, 2006; Olson \& Jiang, 2004). However, despite these efforts, evidence regarding the effects of pre-existing LTM on VSTM has remained mixed (e.g., Brady, Störmer, \& Alvarez, 2016; Quirk, Adam, \& Vogel, 2020; also see Luck, 2008). This uncertainty limits our ability to generalize knowledge of visual cognition from laboratory settings to more natural human behaviors.

To resolve this uncertainty, this current chapter discusses converging evidence in the literature concerning the contribution of pre-existing LTM to the formation of VSTM. Our approach is to identify the conditions under which pre-existing LTM consistently facilitates VSTM. This approach provides a novel context for the development of theories about VSTM, LTM, and their interactions. We will begin with theoretical predictions of the roles of preexisting LTM in VSTM formation, followed by converging evidence from behavioral and neural data in support of some of these predictions. Finally, we will discuss mechanisms underlying these empirical observations and propose some open questions for future investigations.

\section{Potential impacts of pre-existing LTM on VSTM formation}

The formation of VSTM representations involves encoding and converting fragile sensory inputs into durable VSTM representations that can survive new sensory disruption (e.g., saccadic eye movements). Conventionally, encoding is referred to as the registration of sensory inputs, starting from stimulus presentation until its sensory trace dissipates (Cappiello \& Zhang, 2016; Sperling, 1960). An additional process is required to stabilize representations of these fragile sensory inputs, which is often referred to as VSTM consolidation (Jolicoeur \& Dell'acqua, 1998; Vogel et al., 2006; Xie \& Zhang, 2017b). This VSTM consolidation process deploys the focus of attention to a small amount of information to form robust mnemonic representations that can survive interruptions coming from masking or additional sensory inputs during memory maintenance (Jolicoeur \& Dell'acqua, 1998; Jiang, 2004; Vogel et al., 2006; Ricker, Nieuwenstein, Bayliss, \& Barrouillet, 2018). While encoding and consolidation are conceptually different, the distinction between these two is often not explicit in empirical observations (also see a discussion in Ricker \& Cowan, 2014). Therefore, in this current chapter, we refer to VSTM encoding and consolidation as processes of VSTM formation. 
By one estimate (Vogel et al., 2006), VSTM consolidation can rapidly complete within a few hundred milliseconds following stimulus presentation. This estimation was achieved by using a modified change detection task, in which random-pattern masks were inserted at various time points following VSTM stimulus presentation during a brief retention interval (Vogel \& Luck, 2006, also see Turvey 1973). In this paradigm (Figure 1A), observers first try to remember a memory display of a few items. The formation of VSTM for these items is then interrupted by a brief stimulus presentation with random-pattern masks presented at variable levels of memory-and-mask stimulus onset asynchrony (SOA). Because stimulus presentation duration is held constant in this task, the inserted masks primarily disrupt, if not fully terminate (Nieuwenstein \& Wyble, 2014), the post-sensory encoding and consolidation processes. As the SOA between memory and mask displays increases (Figure 1B), the numbers of encoded/consolidated VSTM representations increase with the duration lapsed after stimulus onset, until the total number of VSTM items an observer can eventually remember is reached, which is often referred to as VSTM storage capacity (Cowan, 2001). After this asymptote, the total number of encoded VSTM representations stays at capacity over a short delay period (Bundesen, 1990; Cowan, 2001; Luck \& Vogel, 1997; Miller, 1956; Vogel et al., 2006; but see Jannati, McDonald, \& Di Lollo, 2015 for a summary of an alternative view), unless it is further affected by other factors or processes, such as maintenance and rehearsal (Bayliss et al., 2015; Woodman \& Vogel, 2005).
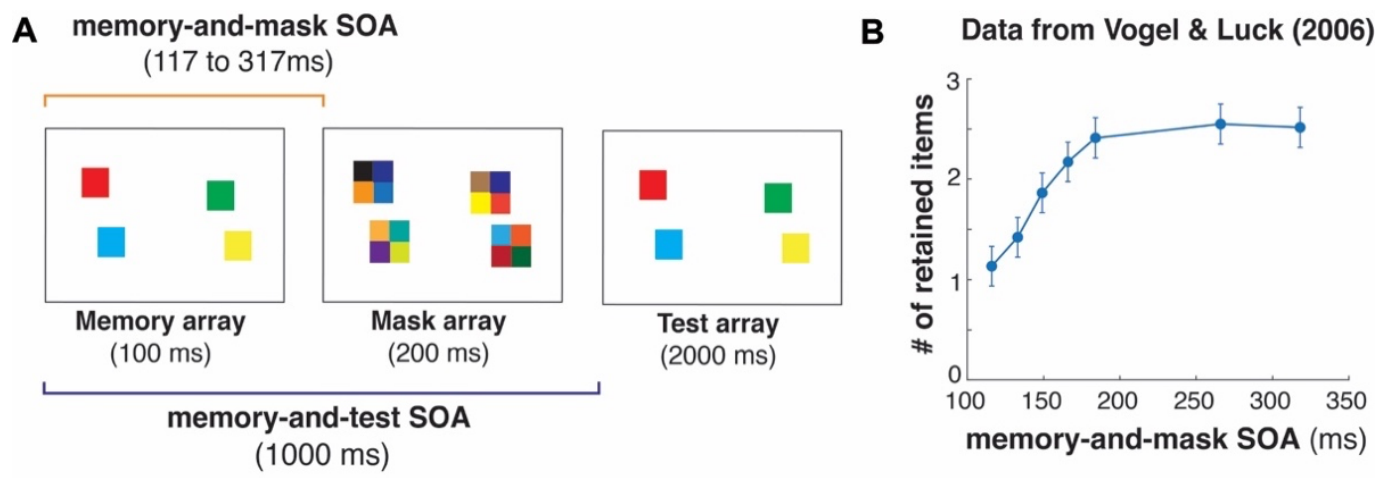

Figure. 1. VSTM change detection paradigm with masking manipulation reveals the time course of VSTM formation. (A) An example trial of the VSTM change detection task with random-pattern masking during a brief VSTM delay period. (B) Data from Vogel and Luck (2006). Each data point represents the mean estimate of the number of remembered VSTM items, and error bars represent $95 \%$ confidence intervals as described by Loftus and Masson (1994). This figure was reproduced from Figure 3 in Vogel and Luck (2006).

Empirically, the speed of VSTM consolidation is under the influences of various topdown factors, such as attention (Vogel \& Luck, 2002), task relevance (Woodman \& Vogel, 2008), and the observer's mental health (Fuller et al., 2005). Based on these empirical observations along with some previous studies on verbal short-term memory (e.g., Mewhort et al., 1969; Phillips, 1971), recent research has proposed that pre-existing LTM as a top-down factor can also modulate the speed of VSTM consolidation (e.g., Blalock, 2015; Xie \& Zhang, 2017b). This 
hypothesis highlights that VSTM formation, especially for more naturalistic visual information, requires both processing visual features of a visual stimulus and situating the stimulus within its LTM representation. This hypothesis makes several key predictions on how pre-existing LTM would affect VSTM over time.

First, by situating a stimulus within its associated pre-existing LTM, observers may rapidly form an internal representation of the stimulus, manifested as faster VSTM consolidation speed. This consolidation speed account predicts that stimuli that the observers have sufficient knowledge about or are more familiar with should be encoded or consolidated into STM with a steeper memory formation slope when the numbers of encoded VSTM items are measured across time (Figure $2 \mathrm{~A}$ and $2 \mathrm{~B}$ ). Alternatively, pre-existing LTM may simply enhance perceptual processing of visual information (Chase \& Simon, 1973; Gobet et al., 2001), hereafter referred to as the perceptual boost account, and subsequently increase the likelihood of encoding the perceptual representation into VSTM in an additive manner (Figure 2C and 2D). This will manifest as a relatively constant increase in the number of retained VSTM items over time within a brief consolidation time window (Sun et al., 2011). This addictive effect can result from various mechanisms, including enhanced holistic encoding (Curby et al., 2009) or chunking (Cowan, 2001) of information due to pre-existing LTM. Conceptually, these effects are independent of LTM's impacts on VSTM capacity, considering the theoretical and empirical dissociation between VSTM consolidation speed and capacity (Bundesen, 1990; Cowan, 2001; Luck \& Vogel, 1997; Miller, 1956; Vogel et al., 2006; but see Jannati, McDonald, \& Di Lollo, 2015). It is thus possible that pre-existing LTM may or may not affect the total number of items one can remember in a VSTM task (i.e., Figure $2 A$ vs. $2 B, 2 C$ vs. $2 D$ ), given that VSTM storage may be limited by the focus of attention at any given moment beyond the influence from preexisting LTM (Cowan, 2001; Oberauer, 2002, 2009).

Two additional issues should be considered for these predictions. First, despite their conceptual independence, the predicted effects of pre-existing LTM on VSTM formation and storage capacity are not mutually exclusive (e.g., Ngiam et al., 2019; Xie \& Zhang, 2017b). Theoretically, a difference in the number of remembered items between familiar and unfamiliar VSTM task stimuli can emerge when VSTM formation is interrupted, either by consolidation masking or insufficient stimulus presentation of the memory items (indicated by the black vertical lines in Figure 2). Hence, the mere presence of a difference in VSTM task performance for familiar and unfamiliar task stimuli may not distinguish whether pre-existing LTM boosts VSTM storage capacity or facilitates VSTM formation. If pre-existing LTM primarily facilitates VSTM formation, VSTM storage capacity may not be influenced by observers' pre-existing LTM of the task stimuli when given sufficient encoding time (Quirk et al., 2020; but see Brady et al., 2016). Second, the predicted facilitation of pre-existing LTM on VSTM does not preclude the possibility that LTM can sometimes hinder VSTM task performance. For example, in a scene change detection task, observers may find it harder to identify a changed item in a test scene if this item is semantically consistent with other items in the scene (e.g., Hollingworth \& Henderson, 2000). This associative LTM effect on VSTM change detection is different from the current focus on pre-existing LTM and VSTM formation at the item level. 
A

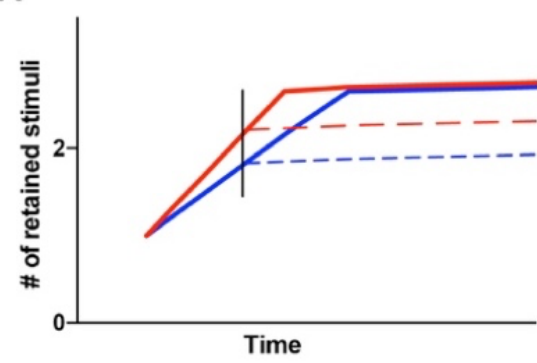

B

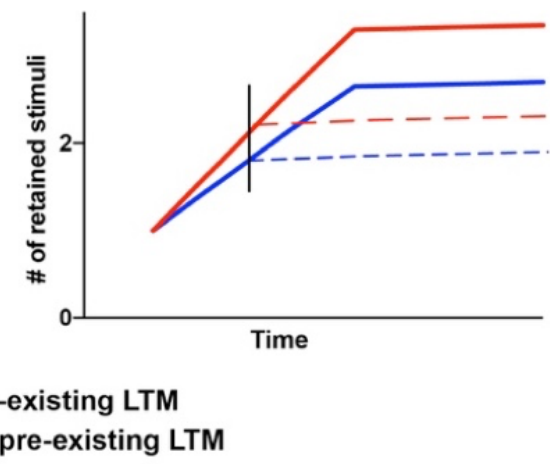

C

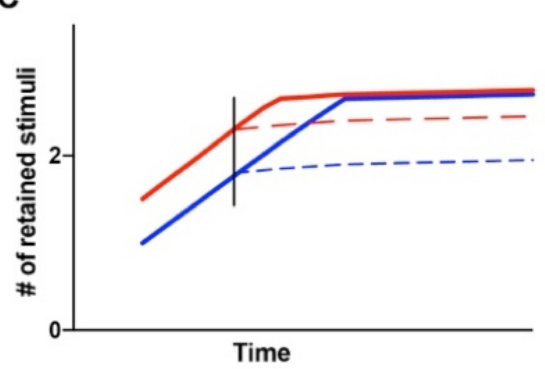

D

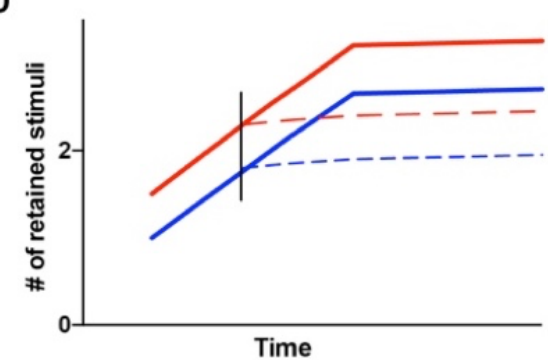

Figure. 2. Potential impacts of pre-existing LTM on VSTM formation. Hypothetically, over the VSTM formation process, the number of retained VSTM representations increases until it plateaus at VSTM capacity. (A, B) The consolidation speed account predicts faster VSTM consolidation (steeper slope) for stimuli with pre-existing LTM, as compared with those without. (C, D) The perceptual boost account predicts that advantages in perceptual processing of stimuli with pre-existing LTM should add a constant boost to VSTM performance over the consolidation process, without affecting VSTM consolidation speed. For both accounts, more information (asymptotes of dashed line) can be encoded into VSTM when the VSTM formation process is interrupted with consolidation masks or insufficient presentation duration of the memory array (indicated by the black vertical lines). This figure is adapted from Figure 1 of Xie and Zhang (2017b).

\section{Evidence that pre-existing LTM facilitates VSTM formation}

A direct test of these different predictions requires estimating the number of items an observer remembers over the course of VSTM formation. As previously introduced, this can be achieved by using the adapted change detection task with masking manipulation (Vogel et al., 2006). In addition to this method, another approach is to assess how the formation of VSTM influences ongoing mental processes during a rapid serial visual presentation (RSVP). This approach is built upon the premise that VSTM formation is a fast but demanding process that occupies the focus of attention (Raffone et al., 2014; Ricker \& Sandry, 2018). Consequently, the consolidation of an item into VSTM may slow down the execution of other cognitive processes that require attention (Jolicoeur \& Dell'acqua, 1998; Nieuwenstein \& Wyble, 2014; Stevanovski \& Jolicœur, 2007). This competition can be revealed by using RSVP, during which participants are asked to detect two sequentially presented salient targets, namely T1 and T2, separated by different numbers of distractors or lags in between (Figure 3A). Participants often fail to detect T2 when it appears within 200-600 ms after the presentation of T1 (Figure 3B), a phenomenon termed as "attentional blink" (Raymond et al., 1992). One hypothesis for this phenomenon is 
that T1 needs to be consolidated into VSTM to support successful detection, which prevents subsequent attention deployment to T2 (Chun \& Potter, 1995; Martens \& Wyble, 2010). Alternatively, this effect may reflect the competition between the selection of T2 and the inhibition of distractors in a time-dependent manner (see a review in Dux \& Marois, 2009). These mechanisms in part reflect the temporal limit in VSTM formation, if they are not directly related to the mechanism for VSTM consolidation.
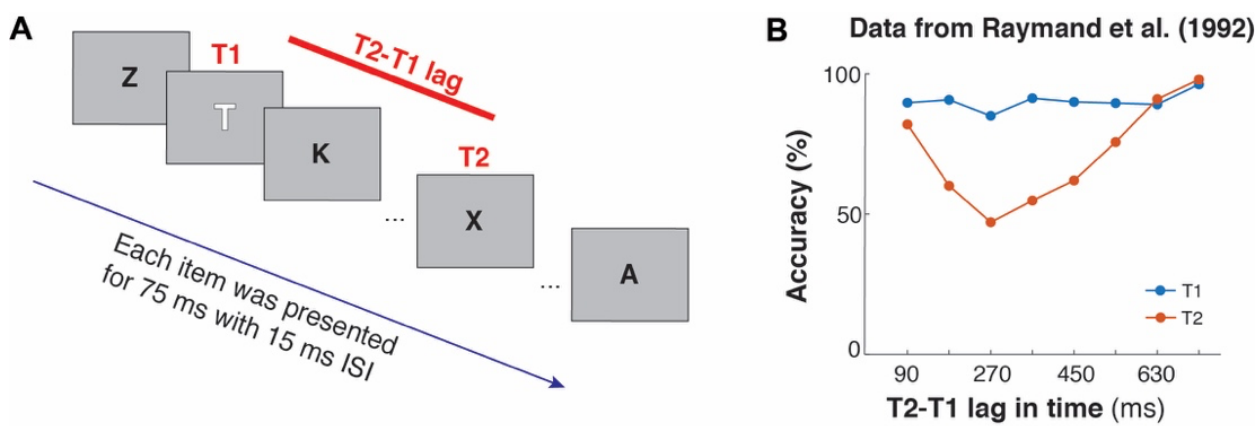

Figure. 3. Attentional blink paradigm can also be used to infer the effect of VSTM formation on subsequent task performance. (A) An example trial of the attentional blink task with both Target 1 and 2 (T1 \& T2). T1 is the letter "T" marked in white font, and T2 is the letter "X." Observers are asked to respond to both T1 and T2 if they are present (dual-task condition) or only to T2 (single-task condition). (B) The attentional blink effect is revealed by the reduction in task performance in $\mathrm{T} 2$ detection accuracy within a certain amount of time after the successful detection of T1. This figure is adapted from Figure 3 in Raymond et al. (1992).

Using these paradigms, converging evidence has emerged to support the hypothesis that pre-existing LTM boosts VSTM formation. Although these experimental approaches have their pros and cons in revealing the process of VSTM formation (see some discussions in Ricker \& Sandry, 2018), the convergence of empirical observations suggests the robustness of the discussed phenomenon.

\subsection{Pre-existing LTM speeds up VSTM formation}

One line of research has used the masking paradigm (Vogel et al., 2006) to show that pre-existing LTM speeds up VSTM formation (Blalock, 2015; Ngiam et al., 2019; Xie \& Zhang, 2017b). These studies operationalize participants' pre-existing LTM for VSTM task stimuli in different ways. For example, Blalock (2015) trained observers to remember novel polygons that presumably are meaningless to the observers, and then tested observers' VSTM task performance for the trained polygons as compared with novel polygons. Blalock (2015) included a range of memory-and-mask SOA, from 517 ms to 984 ms, given a fixed 500-ms stimulus presentation. Blalock (2015) found that participants' VSTM performance for trained items already reached asymptote and was unaffected by visual masks at the earliest memoryand-mask SOA of $517 \mathrm{~ms}$, whereas their performance for novel polygons did not peak until a much later memory-and-mask SOA of 867 ms. Blalock (2015) therefore concluded that trained polygons were consolidated faster into VSTM. This study also found that the early facilitation of VSTM for trained polygons persisted throughout VSTM maintenance. However, because VSTM 
consolidation could take place or even have already been completed during the $500-\mathrm{ms}$ stimulus presentation, both boosted VSTM consolidation or perceptual encoding (Figure 2) can account for the observations by Blalock (2015).

To resolve this issue, Xie and Zhang (2017b) thus used shorter stimulus presentation duration (100 ms) and shorter memory-and-mask SOA (117-ms vs. 333-ms) to examine whether pre-existing LTM speeds up VSTM formation or boosts perceptual encoding. In this study, participants were asked to remember a set of cartoon characters from Pokémon, a media and game franchise that gains global popularity in recent decades stimuli, in a VSTM task with masking manipulation (Figure 4A). Participants' pre-existing LTM therefore was manipulated based on their prior multimedia experiences with Pokémon. The first-generation Pokémon was released in 1998, with multiple subsequent releases of new Pokémon characters with comparable style and complexity in the past two decades. College participants with prior exposure to the Pokémon franchise tend to have more experience with older generations of Pokémon, but have less experience with recent ones (e.g., the fifth generation was released in 2011). In contrast, those who are not familiar with the Pokémon franchise tend to be unfamiliar with both the first and recent generations of Pokémon. Thus, observers' natural multimedia exposure of Pokémon offers an opportunity for both within-subject (e.g., first vs. recent generation Pokémon) and between-subject (e.g., high vs. low familiarity level with Pokémon at the subject level) comparisons of VSTM task performance under the influence of pre-existing LTM of Pokémon. To support these comparisons, in a separate study, Xie and Zhang (2017b) verified that observers' familiarity with Pokémon indeed captured their prior experiences with Pokémon, based on the consistency between self-report familiarity and the accuracy in a Pokémon knowledge quiz (also see Gomez, Barnett, \& Grill-Spector, 2019).

By comparing the number of encoded Pokémon items between 117-ms and 333-ms memory-and-mask SOA conditions, Xie and Zhang (2017b) found that first-generation Pokémon characters that participants were more familiar with were consolidated faster into VSTM, as compared with recent-generation Pokémon characters that participants were less familiar with (Figure 4B, Left). These effects were absent in participants who were unfamiliar with both generations of Pokémon (Figure 4C, Left). Additionally, when no mask was inserted in between memory and test (i.e., no-mask condition), they found that the Pokémon familiarity could increase the number of retained Pokémon stimuli when consolidation was uninterrupted but still incomplete due to insufficient encoding time (100 ms). However, when encoding time in the no-mask condition increased to $1,000 \mathrm{~ms}$ in a subsequent experiment, they found that Pokémon familiarity could no longer significantly enhance VSTM task performance. These data suggest that pre-existing LTM may not necessarily break the bottleneck of VSTM storage capacity when participants are given sufficient time to encode both familiar and unfamiliar task stimuli. Instead, the increase in VSTM storage capacity for familiar information seems largely an artifact of certain experimental procedures that could interrupt VSTM formation (e.g., the vertical lines in Figure 2). These findings therefore reveal a key moderator moderating the presence and absence of LTM-related VSTM capacity effects. Considering this moderator and its related individual differences may thus provide a better context to interpret some mixed findings in the literature (e.g., Quirk et al., 2020; Brady et al., 2016). 
A $\begin{gathered}\text { memory-and-mask SOA } \\ (117 \mathrm{~ms} \text { or } 333 \mathrm{~ms} \text { or no-mas })\end{gathered}$
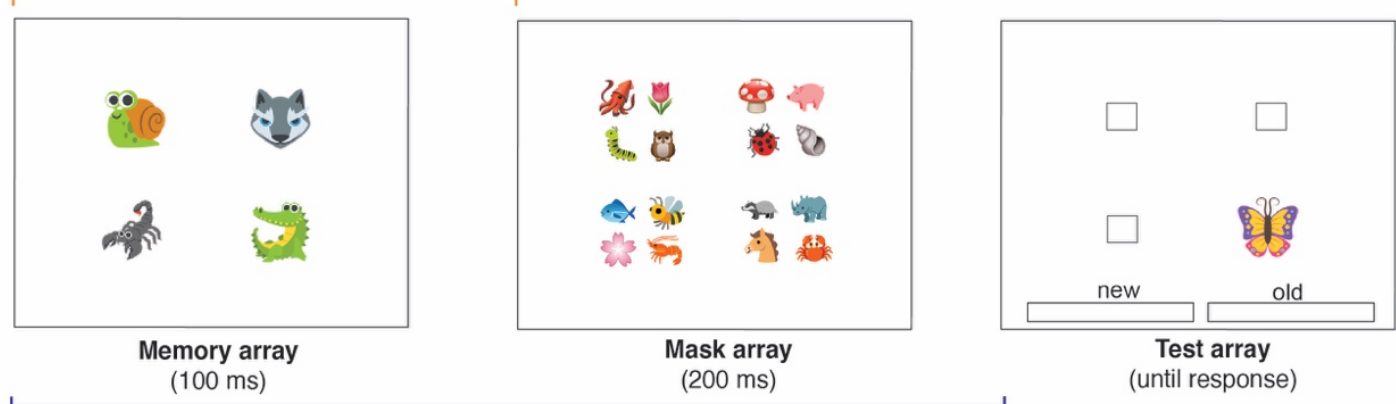

memory-and-test SOA

(1600 ms)

B
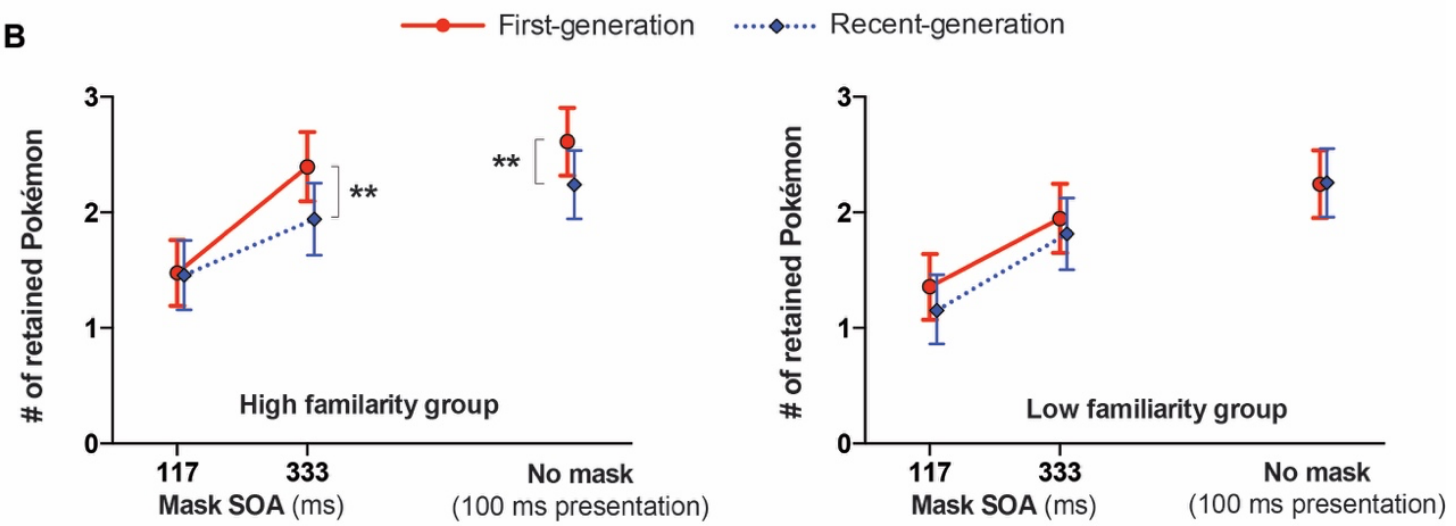

Figure. 4. Experimental task and ERP results from Xie and Zhang (2017b). (A) An example trial of the Pokémon change detection task with random-pattern masking during the delay period. Each random-pattern mask is composed of four smaller Pokémon items that cover the visual area of a memory item. There is no overlap between memory items and mask items. Here, Pokémon stimuli are replaced with open-sourced emojis, emojipedia.org, for copyright protection of Pokémon images. (B) Numbers of retained Pokémon characters across masking and no-mask conditions for high-familiarity (left) and low-familiarity participants (right). Error bars indicate within-subject 95\% confidence intervals (Morey, 2008). **. $p<.01$. This figure was adapted from Figure 3 in Xie and Zhang (2017b).

A recent study (Ngiam et al., 2019) replicated these findings based on familiar stimuli of English letters and unfamiliar artificial letters from the Brussels Artificial Character Set (BACS). The BACS is a set of designed stimuli that share the same perimetric complexity as well as the same number of junctions, strokes, and terminations as English letters (Vidal et al., 2017; Figure 5A). Most English speakers are unfamiliar with these stimuli. The study by Ngiam and colleagues (2019) took advantage of these stimuli to isolate the contribution of visual familiarity to the VSTM of English letters from stimulus complexity, affirming prior observations using a similar approach (e.g., Mewhort et al., 1969; Phillips, 1971). 
A

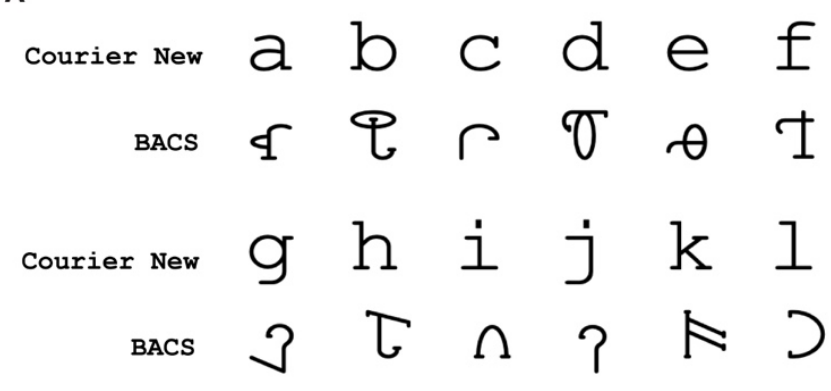

B

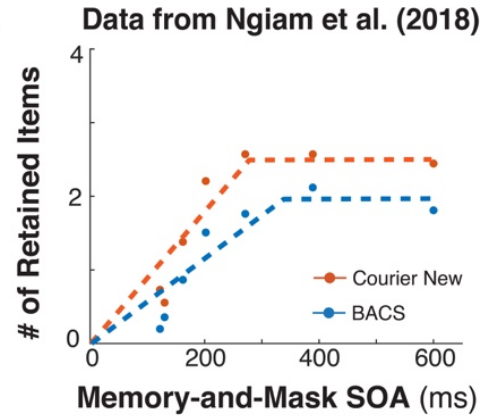

Figure. 5. Stimuli and observations from Ngiam et al. (2019). (A) Example letters from the BACS are compared with corresponding regular letters in Courier New font. These stimuli are visually and quantitatively similar with one another in terms of perimetric complexity. (B) Yet, when presented for 100-ms, regular letters in Courier New font are encoded/consolidated into VSTM much faster as compared with BACS letters. Observers also remember more regular letters than BACS letters at long SOA conditions, given a short stimulus presentation duration of 100 ms. This figure is adapted from Figure 11 in Ngiam et al. (2019). Each data point represents mean number of retained VSTM items at each experimental condition. The dashed lines represent model fit results from the study.

In this study, Ngiam et al. (2019) presented the memory items with a brief presentation duration of $100 \mathrm{~ms}$. Afterward, they inserted a random-pattern mask at memory-and-mask SOA of $120,130,160,200,270,390$, or 600 ms in different experimental conditions. The brief stimulus presentation with a wide range of memory-and-mask SOAs allows them to better reveal the time course of VSTM formation. They found that the number of encoded English letters increased more rapidly with time, as compared with BACS stimuli (Figure 5B), before it reached asymptote. Similar to the no-mask condition in Xie and Zhang (2017b), Ngiam et al. (2019) also found that observers remembered more items for familiar English letters relative to unfamiliar BACS stimuli at long SOA conditions. Given the short stimulus presentation of 100 $\mathrm{ms}$, it is likely that VSTM formation is incomplete even at these long memory-and-mask SOAs (e.g., see vertical black lines in Figure 2). It is thus important to further examine whether the disadvantage for the VSTM of BACS stimuli as compared with regular English letters would disappear under a longer encoding time (Quirk et al., 2020).

Collectively, using the masking paradigm, converging evidence based on different task stimuli and experimental procedures has suggested that pre-existing LTM speeds up VSTM formation (Blalock, 2015; Ngiam et al., 2019; Xie \& Zhang, 2017b). The effect of pre-existing on VSTM storage capacity, however, may be complicated by other factors such as the presentation duration of memory stimuli (Xie \& Zhang, 2017a, 2017b). While these findings support the prediction depicted by Figure $2 \mathrm{~A}$, several caveats are noted when interpreting these results. First, semantic information may have a different contribution to VSTM formation as compared with visual familiarity. For example, using semantic stimuli, Sun and colleagues (2011) observed different findings as compared with Ngiam et al. (2019), when they contrasted Chinese and German observers' VSTM formation for Chinese characters relative to novel and meaningless symbols. Sun et al. found that familiar items primarily increased VSTM performance equally across time for VSTM formation, suggesting that familiarity boosted the likelihood of VSTM encoding without speeding up VSTM consolidation (Figure 2B). One critical difference between Ngiam et al. and Sun et al. is that a Chinese character is a higher-level semantic unit as 
compared with an English letter. A Chinese character has concrete a semantic meaning that corresponds more closely to a word in English (Zimmer \& Fischer, 2020). This additional semantic involvement may recruit more semantic encoding beyond visual familiarity. Second, while the studies discussed here included an articulatory suppression procedure during the VSTM task to interrupt explicit verbal encoding of the task stimuli (Besner, 1987), this procedure does not fully block verbal processing (Norris et al., 2018). Although the complex visual stimuli used in these studies (e.g., polygons and Pokémon) are sometimes harder to name or label as compared with letters or Chinese characters, these studies could not completely rule out semantic contributions to VSTM formation (also see later discussions).

\subsection{Pre-existing LTM reduces attentional blink}

Another line of research based on the attentional blink paradigm also supports the prediction that pre-existing LTM speeds up VSTM formation. The foremost observation is that pre-existing LTM about a target can attenuate attention blink, leading to higher accuracy for T2 at short versus long T1-T2 lags. For example, in an RSVP study, Burt and colleagues (2011) asked observers to detect two words (T1 and T2) among non-word distractors. They manipulated participants' familiarity with T1 based on word frequency, after factoring out the orthographic similarity of the words. Across experiments, Burt and colleagues (2011) found that observers' successful detection of a familiar T1 attenuated the attentional blink effect for T2, regardless of the familiarity level of T2 (Figure 6A). This observation was replicated by Wierda and colleagues (2013). Consistent with the consolidation account for attention blink, these findings suggest that pre-existing LTM about T1 speeds up its consolidation into VSTM, more rapidly freeing up attention resources for later processing of T2 (Chun \& Potter, 1995; Martens \& Wyble, 2010).

Does the effect of pre-existing LTM on VSTM formation also directly affect T2 detection when the familiarity level of T1 is held constant? In an RSVP, Jackson and Raymond (2006) asked participants to first judge the texture of a novel T1 image and then try to detect a prespecified face (T2) presented at varying lags after T1 among other faces as distractors. They manipulated participants' familiarity with face stimuli by using famous familiar or novel unfamiliar faces in this study. They found that the detection of more familiar T2 faces was significantly better at short T1-T2 lags as compared with less familiar faces (Figure 6B). This effect exists regardless of observers' familiarity levels of the distractor faces. Jackson and Raymond's (2006) results were replicated in various studies using personally familiar faces (Gobbini et al., 2013), objects of expertise (Blacker \& Curby, 2016), familiar scene images (Guilbert et al., 2020), and familiar audio stimuli (Akça et al., 2020), indicating the robustness of this phenomenon.

Collectively, these observations highlight that pre-existing LTM influences the temporal limit in processing multiple items within a brief VSTM consolidation time window (Chun \& Potter, 1995; Martens \& Wyble, 2010). This influence can manifest in two different ways. First, as a familiar stimulus can be rapidly consolidated into VSTM, deployment of attention resource to this stimulus (T1) would not occupy all the resources for the processing of a subsequent 
stimulus (T2) within a brief consolidation time window. Consequently, the attentional blink effect for T2 is attenuated following the detection of a familiar T1 (Burt et al., 2011; Wierda et al., 2013). Second, even when initial visual processing is under the influence of an unfamiliar stimulus (T1), the processing of a subsequent stimulus (T2) will also be more fluent when this stimulus is familiar to the observer (Jackson and Raymond, 2006). However, as noted by Wierda et al. (2013), this second scenario may be more complex when we consider the interaction between observers' familiarity levels of T1 and T2. Furthermore, VSTM consolidation limit may not be the only mechanism underlying attentional blink (see a review in Dux \& Marois, 2009). It is also possible that pre-existing LTM may affect other factors, such as freeing up task load to facilitate distractor inhibition, to support the successful detection of T2. Future research needs to further evaluate this possibility.
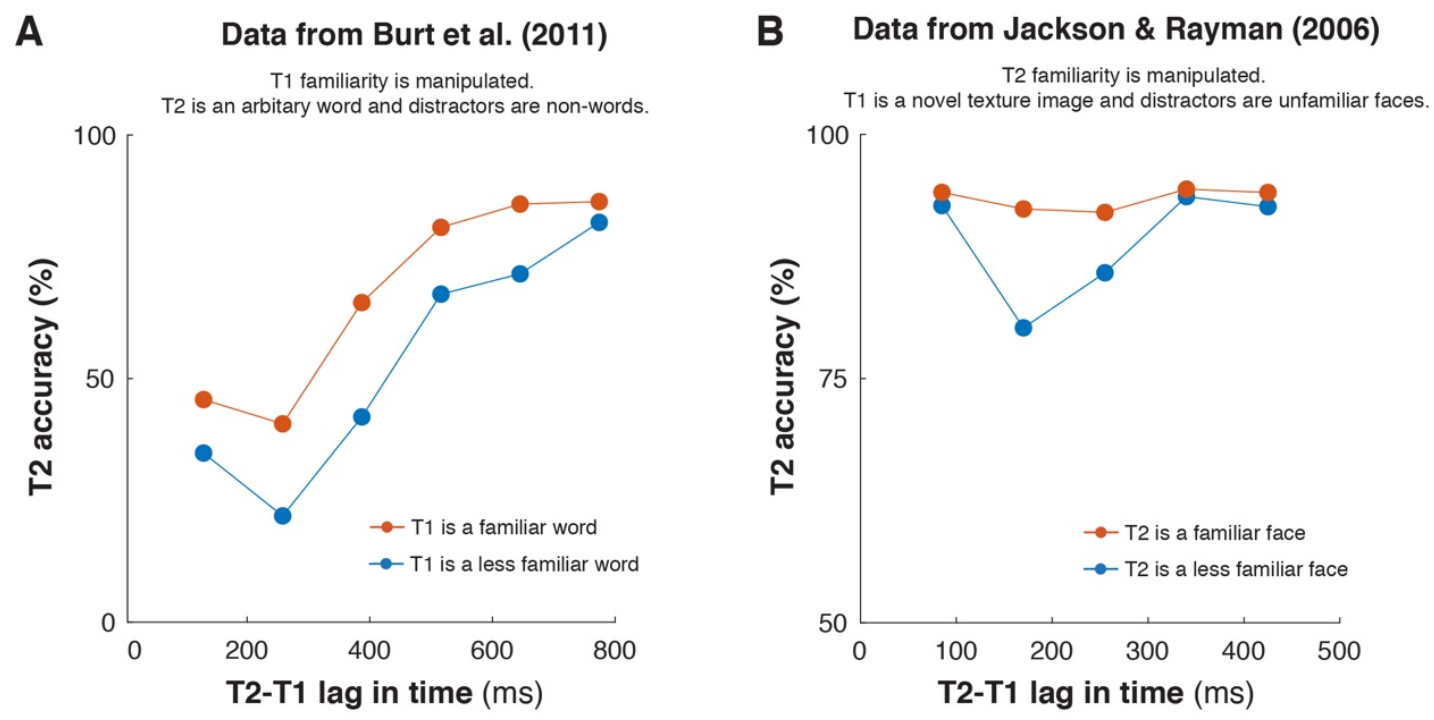

Figure. 6. Observers' pre-existing LTM with task stimuli attenuate attentional blink. (A) Observations from Burt et al. (2011) showing reduced attentional blink effect when T1 is a familiar high-frequency word and T2 is an arbitrary word with variable word frequency and orthographic similar level, among non-word distractors. This figure is adapted from Figure 6 in Burt (2011). (B) Observations from Jackson and Raymond (2006) showing reduced attentional blink effect when $\mathrm{T} 1$ is an arbitrary texture image and $\mathrm{T} 2 \mathrm{is}$ a familiar face, while distractors are unfamiliar faces. This figure is adapted from Figure 5 in Jackson and Raymond (2006). Each data point represents mean $\mathrm{T} 2$ accuracy at each experimental condition when $\mathrm{T} 1$ is correctly detected.

\subsection{Neurophysiological evidence related to boosted VSTM formation with pre-existing LTM}

Both masking and RSVP manipulations interrupt VSTM processing so that behavioral effects associated with VSTM formation can be observed. However, these manipulations could also introduce additional effects on processes other than VSTM formation (Ricker \& Sandry, 2018; Xie \& Zhang, 2018). To resolve this issue, neurophysiological methods such as ERPs can be used to directly measure VSTM formation. 
Specifically, the ERP component of contralateral delay activity (CDA) is of interest. Introduced by Vogel and Machizawa (2004), CDA is a sustained negative deflection over parietal-occipital electrodes based on scalp electroencephalogram during the VSTM delay period. To elicit CDA, in a VSTM task with a bilateral display, observers are asked to remembered stimuli in one visual field as indicated by a cue before stimulus onset, while maintaining the gaze at the center of the screen. As observers pay more attention to one side of the visual field than another side, ERP waveform contralateral to the cued side tends to show more negative deflection as compared with ERP waveform ipsilateral to the cued side. Because stimulus-level features (e.g., set size, luminance, and complexity) are matched across the visual fields, the difference in ERP waveform between hemispheres, namely the CDA, primarily reflects the neural processes for the retention of task-relevant VSTM content. Furthermore, as the CDA is measured from the delay interval prior to any response, it is void of the impact from any retrieval and decisional factors occurring after the onset of the test display.

Several additional attributes of the CDA make it suitable to reveal how pre-existing VSTM affects VSTM formation. First, the amplitude of CDA increases over time, reflecting the increase in the amount of information encoded into STM for both simple and complex stimuli (Fukuda, Awh, \& Vogel, 2010). Changes in CDA amplitude during the rising phase are therefore indicative of the speed of VSTM formation within a given encoding duration (Qi et al., 2014). Second, CDA amplitude during the late phase of VSTM retention increases as a function of VSTM set size and plateaus at a memory set size consistent with behavioral estimates of STM storage capacity (Vogel \& Machizawa, 2004). CDA amplitude captured at an earlier and a later period of a VSTM task may thus reflect VSTM formation and storage capacity, respectively. Finally, complementary to these amplitude measures, the time it takes for CDA amplitude to rise at a certain percentage of its maximum amplitude - namely fractional area latency - also increases linearly from one to four memory items until it reaches VSTM capacity (Perez \& Vogel, 2012). This temporal sensitivity of both CDA amplitude and latency is advantageous to reveal experimental impacts on VSTM representations and processes over time.

Xie and Zhang (2018) leveraged these features of the CDA in a Pokémon VSTM change detection task to evaluate the impacts of pre-existing VSTM on VSTM formation without masking interruption under more sufficient encoding time (1,000 ms; Figure 7A). They found that both the early-phase CDA amplitude and the CDA 50\% fractional area latency were significantly associated with individual differences in Pokémon familiarity. Participants who were more familiar with Pokémon showed faster CDA modulation for the first-generation Pokémon, as compared with unfamiliar recent-generation Pokémon (Figure 7B). This observation was not seen in those who had with minimal Pokémon familiarity (Figure 7C). 
A memory-and-test SOA
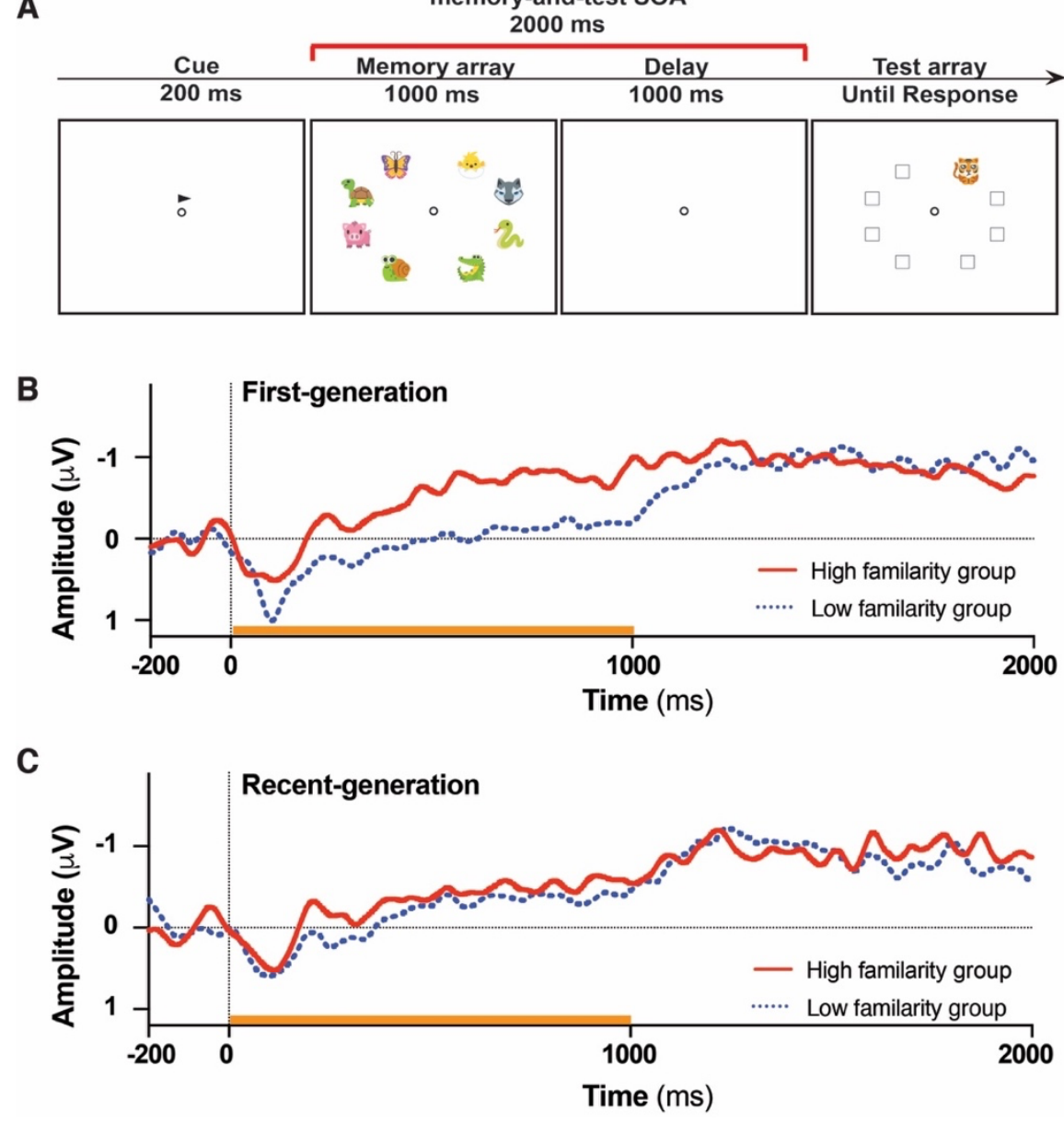

Figure. 7. Experimental task and ERP results from Xie and Zhang (2018). (A) An example trial of the Pokémon change detection with lateralized attention cuing for the measurement of CDA. Participants are asked to remember the Pokémon stimuli presented in the visual field indicated by an arrow cue, while maintaining the eye gaze at the center of the screen. Here, Pokémon stimuli are replaced with open-sourced emojis, emojipedia.org, for copyright protection of Pokémon images. The grand-averaged CDA waveforms for the high- and low-familiarity subject groups are plotted separately for first-generation (B) and recent-generation (C) Pokémon. The stimuli presentation duration is marked as orange on the $\mathrm{x}$-axis of $(\mathbf{B})$ and $(\mathbf{C})$. This figure is adapted from Figures 1 \& 3 in Xie \& Zhang (2018).

Furthermore, because participants were given a sufficiently long memory stimulus duration $(1,000 \mathrm{~ms})$, they were able to remember comparable numbers of Pokémon items across stimulus familiarity in the end, despite the difference during the VSTM formation period. This observation was supported not only by behavioral estimates of the numbers of remembered Pokémon given a 1,000-ms encoding time (also see Xie \& Zhang, 2017b), but also by a comparable level of CDA amplitude at the later phase of the VSTM retention period for both familiar and unfamiliar Pokémon stimuli (Figure 7B and 7C). Consistent with previous findings, stimulus familiarity could lead to more remembered Pokémon items and a higher latephase CDA amplitude when VSTM formation is incomplete due to reduced presentation duration $(500 \mathrm{~ms})$ in their second experiment. These data further support the interpretation 
that faster VSTM formation for familiar stimuli may or may not evolve into a capacity effect, based on whether the VSTM consolidation process is interrupted or not.

Similar observations have also been found in a recent study by Asp and colleagues (2021), in which they manipulated the influences of pre-existing LTM on VSTM using Mooney stimuli (Mooney, 1957), which are seemingly random black-and-white ink bolts. These stimuli are often well-matched in visual complexity, and some could be recognized as meaningful objects like faces. When observers identify a face in a Mooney image, it means that they assign some meanings to the stimulus, presumably from their pre-existing LTM. Asp et al. (2021) found that when observers identified more faces in a lateralized visual display of Mooney images, participants tend to show a larger CDA amplitude, which emerged while the stimulus was still presented on the screen and persisted through the last $500 \mathrm{~ms}$ before response (see Figure 4C in Aps et al., 2021). Asp and colleagues therefore concluded that meaningful stimuli might enhance temporary VSTM storage, potentially by recruiting additional neural mechanisms to retain task-relevant information (e.g., fusiform face area for faces). As noted by Asp et al., however, stimulus meaningfulness and visual familiarity may reflect different types of pre-existing LTM. To some extent, Asp et al.'s observations are similar to the findings by Sun et al. (2011), in that the meanings of task stimuli (e.g., faces or Chinese characters) may boost perceptual encoding of task content, even before VSTM consolidation starts. It is of great research to clarify whether meaningful Mooney faces also speed up VSTM consolidation, potentially by measuring the numbers of encoded Mooney faces over the course of VSTM consolidation using the masking paradigm.

\section{Possible mechanisms and future directions}

Despite robust behavioral and neurophysiological evidence showing the facilitation of pre-existing LTM on VSTM formation, the neural mechanisms underlying this effect remain unknown. Here, we identify three emerging hypotheses for some of these observations. Testing these hypotheses in future studies may promote our understanding of the organization of visual memory and the functional relevance of cognitive training in naturalistic visual cognition.

First, the facilitation of pre-existing LTM on VSTM formation may be grounded in enhanced neural processing of familiar or expertise information represented in association cortices. This hypothesis is supported by the rich literature in neural plasticity for visual items in the ventral stream (e.g., Gauthier et al., 1999; Gomez et al., 2019; James \& James, 2013). For example, James et al. (2013) and Gomez et al. (2019) found that participants' extensive prior visual experiences with Pokémon can lead to distinct cortical responses to Pokémon stimuli in the inferior temporal regions. It is possible that this experience-induced neural plasticity subsequently facilitates the conversion of sensory experiences with Pokémon stimuli into durable VSTM representations. This prediction is partly supported by the observation that inferior temporal regions tend to retain category-specific information during both the encoding and retention period of a VSTM task (e.g., Ranganath, DeGutis, \& D’Esposito, 2004). However, thus far, no direct evidence has linked these inferior temporal signals with speeded-up VSTM 
formation. Future research with recording techniques that have a higher temporal resolution and a reasonable spatial resolution (e.g., magnetoencephalography or intracranial electroencephalogram) may better address this issue.

Second, it is also possible that pre-existing LTM facilitates VSTM formation by making the neural coding of familiar visual information more efficient (Bates \& Jacobs, 2020). Efficiency can be conceptualized, among several possibilities, as the minimal amount of data units required for information coding (Bates \& Jacobs, 2020). For example, Morse code leverages letter frequency in English to optimize its efficiency in converting letters of the alphabet to binary signals ("." and "--"). By assigning shorter binary sequences to more frequently encounter letters, it minimizes the total data units needed for more complex messages, such as phrases, sentences, or paragraphs. The same principle may also be applied to the encoding of VSTM information, such that the visual system may be tuned to some statistical regularity in the environment based on prior experiences (Brady et al., 2009). This process is also akin to chunking where meaningful information is grouped together (Cowan, 2001). While this hypothesis does not imply structural or functional neural changes, it is of great interest to clarify how the optimation of information coding is implemented in the human brain.

Finally, VSTM formation may rely upon not only visual sensory information but also preexisting semantic or conceptual knowledge about the visual stimuli (Potter, 2012). Rich behavioral data suggest that semantic information about an object or scene and its associations can be rapidly accessed as early as 100-300 ms following stimulus presentation (see Potter, 2012 for a summary). A recent study based on direct recording from the human brain with intracranial electroencephalography provides some evidence to support these behavioral observations (Liu et al., 2020). Liu et al. (2020) examined the correlations between neural signals recorded during VSTM formation with visual features of everyday objects derived from a deep neural network (DNN) and with semantic features of these objects. This approach reveals the amount of visual and semantic contributions during VSTM formation. Liu et al. found that both visual and semantic codes of real-life objects play an important role during VSTM formation and retention. A caveat, however, is that higher DNN layers, which presumably reflect higher-order perceptual features, are usually closely correlated with semantic features of the objects. This raises an interesting question about the extent to which visual and semantic contributions can be dissociated during VSTM formation. Future studies with a stimulus set that orthogonalizes higher-order perceptual similarity and semantic similarity of task stimuli may better address this issue (see a discussion in Xie \& Zaghloul, 2021).

\section{Conclusions}

Based on converging behavioral evidence using different experimental paradigms and neural evidence from the CDA, this chapter has demonstrated that pre-existing LTM facilitates VSTM formation. By solidifying this line of research, our approach highlights the importance of pre-existing LTM on the initial VSTM processes beyond perception (e.g., Stokes et al., 2012). This approach is complementary to the research on the effects of LTM on VSTM 
representations, such as the number and precision of retained VSTM contents (e.g., Brady et al., 2016; Lorenc et al., 2014; Quirk et al., 2020; Xie \& Zhang, 2017b). This work thus advances our understanding of the interaction between LTM and VSTM in several important ways.

First, in contrast to the traditional view that VSTM primarily consists of structuredprimitives of visual elements (e.g., colors, orientations, and 3-D volumes, Biederman, 1987), this line of research suggests that the formation of VSTM representations is not immune to LTM influences. Task content that matches more closely with pre-existing LTM tends to be more fluently processed (see a discussion in Luck, 2008). Second, as the impact of pre-existing LTM on VSTM may be time-dependent, probing observers' VSTM task performance too early or too late may only yield a modest experimental effect (Figure 2A). Taking into account the representational limit (e.g., capacity) and the processing limit (e.g., consolidation speed) of VSTM, therefore, may allow us to better assess mixed findings about the training effects on VSTM (e.g., Blalock, 2015; Olson \& Jiang, 2004) and the VSTM storage capacity for real-life objects (e.g., Brady et al., 2016; Quirk et al., 2020). Finally, the effect of pre-existing LTM on VSTM also depends on observers' LTM strength of VSTM task content. It is not surprising that relatively weak LTM representations may not lead to substantial changes in VSTM representations or processes. However, overtrained or super-strong LTM can influence VSTM via multiple mechanisms including perceptual and semantic processes (e.g., Curby et al., 2009; Stokes et al., 2012; Sun et al., 2011). Delineating the impact of pre-existing LTM on VSTM representations or processes therefore requires an awareness of individual differences, especially when we use real-world objects in naturalistic visual cognition. 


\section{References}

Akça, M., Laeng, B., \& Godøy, R. I. (2020). No Evidence for an Auditory Attentional Blink for Voices Regardless of Musical Expertise. Frontiers in Psychology, 10, 2935. https://doi.org/10.3389/fpsyg.2019.02935

Asp, I. E., Störmer, V. S., \& Brady, T. F. (2021). Greater Visual Working Memory Capacity for Visually Matched Stimuli When They Are Perceived as Meaningful. Journal of Cognitive Neuroscience, 1-17. https://doi.org/10.1162/jocn_a_01693

Atkinson, R. C., \& Shiffrin, R. M. (1968). Human memory: A proposed system and its control processes. Psychology of Learning and Motivation, 2, 89-195.

Awh, E., Barton, B., \& Vogel, E. K. (2007). Visual working memory represents a fixed number of items regardless of complexity. Psychological Science, 18(7), 622-628.

Baddeley, A. D. (2012). Working memory: Theories, models, and controversies. Annual Review of Psychology, 63, 1-29. https://doi.org/10.1146/annurev-psych-120710-100422

Bates, C. J., \& Jacobs, R. A. (2020). Efficient data compression in perception and perceptual memory. Psychological Review, 127(5), 891-917. https://doi.org/10.1037/rev0000197

Bayliss, D. M., Bogdanovs, J., \& Jarrold, C. (2015). Consolidating working memory: Distinguishing the effects of consolidation, rehearsal and attentional refreshing in a working memory span task. Journal of Memory and Language, 81, 34-50.

https://doi.org/10.1016/j.jml.2014.12.004

Besner, D. (1987). Phonology, Lexical Access in Reading, and Articulatory Suppression: A Critical Review. The Quarterly Journal of Experimental Psychology Section A, 39(3), 467-478. https://doi.org/10.1080/14640748708401799

Biederman, I. (1987). Recognition-by-components: A theory of human image understanding. Psychological Review, 94(2), 115-147. https://doi.org/10.1037/0033-295X.94.2.115

Blacker, K. J., \& Curby, K. M. (2016). The attentional blink is attenuated for objects of expertise. Visual Cognition, 24(2), 132-140. https://doi.org/10.1080/13506285.2016.1200701

Blalock, L. D. (2015). Stimulus familiarity improves consolidation of visual working memory representations. Attention, Perception \& Psychophysics, 77(4), 1143-1158.

Brady, T. F., Konkle, T., \& Alvarez, G. A. (2009). Compression in Visual Working Memory: Using Statistical Regularities to Form More Efficient Memory Representations. Journal of Experimental Psychology: General, 138(4), 487-502. https://doi.org/10.1037/a0016797

Brady, T. F., Störmer, V. S., \& Alvarez, G. A. (2016). Working memory is not fixed-capacity: More active storage capacity for real-world objects than for simple stimuli. Proceedings of the National Academy of Sciences of the United States of America, 113(27), 7459-7464. Retrieved from http://www.pnas.org/lookup/doi/10.1073/pnas.1520027113

Brady, T. F., Störmer, V. S., Shafer-Skelton, A., Williams, J. R., Chapman, A. F., \& Schill, H. M. (2019). Scaling up visual attention and visual working memory to the real world.

Psychology of Learning and Motivation - Advances in Research and Theory, 70, 29-69. https://doi.org/10.1016/bs.plm.2019.03.001

Bundesen, C. (1990). A theory of visual attention. Psychological Review, 97(4), 523-547.

Burt, J. S., Howard, S., \& Falconer, E. K. (2011). T1 difficulty affects the AB: Manipulating T1 word frequency and T1 orthographic neighbor frequency. Attention, Perception, and Psychophysics, 73(3), 751-765. https://doi.org/10.3758/s13414-010-0054-x

Buttle, H., \& Raymond, J. E. (2003). High familiarity enhances visual change detection for face 
stimuli. Perception \& Psychophysics, 65(8), 1296-1306.

Cappiello, M., \& Zhang, W. (2016). A dual-trace model for visual sensory memory. Journal of Experimental Psychology: Human Perception and Performance, 42(11), 1903-1922.

Chase, W. G., \& Simon, H. A. (1973). Perception in chess. Cognitive Psychology, (4), 55-81.

Chen, D., Yee Eng, H., \& Jiang, Y. (2006). Visual working memory for trained and novel polygons. Visual Cognition, 14(1), 37-54. https://doi.org/10.1080/13506280544000282

Chun, M. M., \& Potter, M. C. (1995). A two-stage model for multiple target detection in rapid serial visual presentation. Journal of Experimental Psychology: Human Perception and Performance, 21(1), 109-127.

Collins, J. A., \& Olson, I. R. (2014). Knowledge is power: How conceptual knowledge transforms visual cognition. Psychonomic Bulletin \& Review, 21(4), 843-860.

Cowan, N. (2001). The magical number 4 in short-term memory: A reconsideration of mental storage capacity. Behavioral and Brain Sciences, 24(1), 87-185.

Cowan, N. (2008). What are the differences between long-term, short-term, and working memory? Progress in Brain Research, 169, 323-338.

Curby, K. M., Glazek, K., \& Gauthier, I. (2009). A Visual Short-Term Memory Advantage for Objects of Expertise. Journal of Experimental Psychology: Human Perception and Performance, 35(1), 94-107. https://doi.org/10.1037/0096-1523.35.1.94

Dux, P. E., \& Marois, R. (2009). The attentional blink: A review of data and theory. Attention, Perception \& Psychophysics, 71(8), 1683-1700.

Endress, A. D., \& Potter, M. C. (2014). Large capacity temporary visual memory. Journal of Experimental Psychology: General, 143(2), 548-565.

Fukuda, K., Awh, E., \& Vogel, E. K. (2010). Discrete capacity limits in visual working memory. Current Opinion in Neurobiology, Vol. 20, pp. 177-182. https://doi.org/10.1016/j.conb.2010.03.005

Fukuda, K., Vogel, E., Mayr, U., \& Awh, E. (2010). Quantity, not quality: The relationship between fluid intelligence and working memory capacity. Psychonomic Bulletin \& Review, 17(5), 673-679.

Fuller, R. L., Luck, S. J., Braun, E. L., Robinson, B. M., McMahon, R. P., \& Gold, J. M. (2009). Impaired Visual Working Memory Consolidation in Schizophrenia. Neuropsychology, 23(1), 71-80. https://doi.org/10.1037/a0013854

Fuller, R. L., Luck, S. J., McMahon, R. P., \& Gold, J. M. (2005). Working Memory Consolidation Is Abnormally Slow in Schizophrenia. Journal of Abnormal Psychology, 114(2), 279-290.

Gauthier, I., Tarr, M. J., Anderson, A. W., Skudlarski, P., \& Gore, J. C. (1999). Activation of the middle fusiform'face area'increases with expertise in recognizing novel objects. Nature Neuroscience, 2(6), 568-573.

Geraldo, A., Azeredo, A., Pasion, R., Dores, A. R., \& Barbosa, F. (2019). Fostering advances to neuropsychological assessment based on the Research Domain Criteria: The bridge between cognitive functioning and physiology. Clinical Neuropsychologist, 33(2), 327-356. https://doi.org/10.1080/13854046.2018.1523467

Gobbini, M. I., Gors, J. D., Halchenko, Y. O., Rogers, C., Guntupalli, J. S., Hughes, H., \& Cipolli, C. (2013). Prioritized Detection of Personally Familiar Faces. PLoS ONE, 8(6), 1-7. https://doi.org/10.1371/journal.pone.0066620

Gobet, F., Lane, P. C. R., Croker, S., Cheng, P. C. H., Jones, G., Oliver, I., \& Pine, J. M. (2001). 
Chunking mechanisms in human learning. Trends in Cognitive Sciences, 5(6), 236-243.

Gomez, J., Barnett, M., \& Grill-Spector, K. (2019). Extensive childhood experience with Pokémon suggests eccentricity drives organization of visual cortex. Nature Human Behaviour, 3(6), 611-624. https://doi.org/10.1038/s41562-019-0592-8

Guilbert, D., Most, S. B., \& Curby, K. M. (2020). Real world familiarity does not reduce susceptibility to emotional disruption of perception: evidence from two temporal attention tasks. Cognition and Emotion, 34(3), 450-461.

https://doi.org/10.1080/02699931.2019.1637333

Hollingworth, A., \& Henderson, J. M. (2000). Semantic informativeness mediates the detection of changes in natural scenes. Visual Cognition, 7(1-3), 213-235.

Jackson, M. C., \& Raymond, J. E. (2006). The role of attention and familiarity in face identification. Perception and Psychophysics, 68(4), 543-557. https://doi.org/10.3758/BF03208757

James, T. W., \& James, K. H. (2013). Expert individuation of objects increases activation in the fusiform face area of children. Neurolmage, 67, 182-192.

Jannati, A., McDonald, J. J., \& Di Lollo, V. (2015). Individual differences in rate of encoding predict estimates of visual short-term memory capacity (K). Canadian Journal of Experimental Psychology/Revue Canadienne de Psychologie Expérimentale, 69(2), 213220.

Jeneson, A., \& Squire, L. R. (2012). Working memory, long-term memory, and medial temporal lobe function. Learning and Memory, 19(1), 15-25.

https://doi.org/10.1101/Im.024018.111

Jiang, Y. (2004). Time Window from Visual Images to Visual Short-Term Memory: Consolidation or Integration? Experimental Psychology, 51(1), 45-51. https://doi.org/10.1027/16183169.51.1.45

Jolicoeur, P., \& Dell'acqua, R. (1998). The demonstration of short-term consolidation. Cognitive Psychology, 36(2), 138-202.

Jonides, J., Lewis, R. L., Nee, D. E., Lustig, C. A., Berman, M. G., \& Moore, K. S. (2008). The Mind and Brain of Short-Term Memory. Annual Review of Psychology, 59(1), 193-224. https://doi.org/10.1146/annurev.psych.59.103006.093615

Karnadewi, F., \& Burt, J. S. (2010). Attentional blink for acronyms: Effects of familiarity and preexposure. Australian Journal of Psychology, 62(3), 130-138. https://doi.org/10.1080/00049530903089521

Klingberg, T. (2010). Training and plasticity of working memory. Trends in Cognitive Sciences, 14(7), 317-324.

Lefton, L. A., \& Spragins, A. B. (1974). Orthographic structure and reading experience affect the transfer from iconic to short-term memory. Journal of Experimental Psychology: General, 103(4), 775-781.

Liu, J., Zhang, H., Yu, T., Ni, D., Ren, L., Yang, Q., ... Xue, G. (2020). Stable maintenance of multiple representational formats in human visual short-term memory. Proceedings of the National Academy of Sciences of the United States of America, 117(51), 32329-32339. https://doi.org/10.1073/pnas.2006752117

Loftus, G. R., \& Masson, M. E. (1994). Using confidence intervals in within-subject designs. Psychonomic Bulletin \& Review, 1(4), 476-490. 
Lorenc, E. S., Pratte, M. S., Angeloni, C. F., \& Tong, F. (2014). Expertise for upright faces improves the precision but not the capacity of visual working memory. Attention, Perception, and Psychophysics, 76(7), 1975-1984. https://doi.org/10.3758/s13414-0140653-z

Luck, S. J. (2008). Visual short term memory. In S. J. Luck \& A. Hollingworth (Eds.), Visual Memory (pp. 43-86). UK: Oxford University Press.

Luck, S. J., \& Vogel, E. K. (1997). The capacity of visual working memory for features and conjunctions. Nature, 390(6657), 279-281.

Martens, S., \& Wyble, B. (2010). The attentional blink: Past, present, and future of a blind spot in perceptual awareness. Neuroscience and Biobehavioral Reviews, 34(6), 947-957. https://doi.org/10.1016/j.neubiorev.2009.12.005

Mewhort, D. J., Merikle, P. M., \& Bryden, M. P. (1969). On the transfer from iconic to shortterm memory. Journal of Experimental Psychology, 81(1), 89-94. https://doi.org/10.1037/h0027467

Miller, G. A. (1956). The magical number seven plus or minus two: Some limits on our capacity for processing information. Psychological Review, 63(2), 81-97.

Mitroff, S. R., Simons, D. J., \& Levin, D. T. (2004). Nothing compares 2 views: Change blindness can occur despite preserved access to the changed information. Perception \& Psychophysics, 66(8), 1268-1281.

Mooney, C. M. (1957). Age in the development of closure ability in children. Canadian Journal of Psychology, 11(4), 219-226. https://doi.org/10.1037/h0083717

Morey, R. D. (2008). Confidence intervals from normalized data: A correction to Cousineau (2005). Tutorial in Quantitative Methods for Psychology, 4(2), 61-64.

Morsh, J. E., \& Stannard, A. F. B. (1947). Studies in international Morse Code. II. A simplified method of determining code speed. Canadian Journal of Psychology Revue Canadienne de Psychologie, 1(2), 67-70. https://doi.org/10.1037/h0084027

Ngiam, W. X. Q., Khaw, K. L. C., Holcombe, A. O., \& Goodbourn, P. T. (2019). Visual working memory for letters varies with familiarity but not complexity. Journal of Experimental Psychology: Learning, Memory, and Cognition, 45(10), 1761-1775. https://doi.org/10.1037/xIm0000682

Nieuwenstein, M., \& Wyble, B. (2014). Beyond a mask and against the bottleneck: Retroactive dual-task interference during working memory consolidation of a masked visual target. Journal of Experimental Psychology: General, 143(3), 1409-1427. https://doi.org/10.1037/a0035257

Norris, D. (2017). Short-Term Memory and Long-Term Memory are Still Different. Psychological Bulletin, 1-19.

Norris, D., Butterfield, S., Hall, J., \& Page, M. P. A. (2018). Phonological recoding under articulatory suppression. Memory and Cognition, 46(2), 173-180. https://doi.org/10.3758/s13421-017-0754-8

Norton, D. J., Parra, M. A., Sperling, R. A., Baena, A., Guzman-Velez, E., Jin, D. S., ... Quiroz, Y. T. (2020). Visual short-term memory relates to tau and amyloid burdens in preclinical autosomal dominant Alzheimer's disease. Alzheimer's Research and Therapy, 12(1), 1-11. https://doi.org/10.1186/s13195-020-00660-z

Oberauer, K. (2002). Access to information in working memory: Exploring the focus of 
attention. Journal of Experimental Psychology: Learning, Memory, and Cognition, 28(3), 411-421.

Oberauer, K. (2009). Design for a Working Memory. In Psychology of Learning and Motivation Advances in Research and Theory (1st ed., Vol. 51). https://doi.org/10.1016/S00797421(09)51002-X

Olson, I. R., \& Jiang, Y. (2004). Visual short-term memory is not improved by training. Memory \& Cognition, 32(8), 1326-1332. https://doi.org/10.3758/BF03206323

Orhan, A. E., \& Jacobs, R. A. (2014). Toward ecologically realistic theories in visual short-term memory research. Attention, Perception, and Psychophysics, 76(7), 2158-2170. https://doi.org/10.3758/s13414-014-0649-8

Perez, V. B., \& Vogel, E. K. (2012). What ERPs can tell us about working memory. In S. J. Luck \& E. S. Kappenman (Eds.), The Oxford Handbook of Event-Related Potential Components (pp. 361-372). University of Oregon, Eugene, United States: Oxford University Press.

Phillips, W. A. (1971). Does familiarity affect transfer from an iconic to a short-term memory? Perception \& Psychophysics, 10(3), 153-157.

Potter, M. C. (2012). Conceptual Short Term Memory in Perception and Thought. Frontiers in Psychology, 3(113), 1-11. https://doi.org/10.3389/fpsyg.2012.00113

Qi, S., Ding, C., \& Li, H. (2014). Neural correlates of inefficient filtering of emotionally neutral distractors from working memory in trait anxiety. Cognitive, Affective, \& Behavioral Neuroscience, 14(1), 253-265.

Quirk, C., Adam, K. C. S., \& Vogel, E. K. (2020). No evidence for an object working memory capacity benefit with extended viewing time. ENeuro, 7(5), 1-13. https://doi.org/10.1523/ENEURO.0150-20.2020

Raffone, A., Srinivasan, N., \& van Leeuwen, C. (2014). The interplay of attention and consciousness in visual search, attentional blink and working memory consolidation. Philosophical Transactions of the Royal Society B: Biological Sciences, 369(1641), 20130215. https://doi.org/10.1098/rstb.2013.0215

Ranganath, C., DeGutis, J., \& D’Esposito, M. (2004). Category-specific modulation of inferior temporal activity during working memory encoding and maintenance. Cognitive Brain Research, 20(1), 37-45. https://doi.org/10.1016/j.cogbrainres.2003.11.017

Raymond, J. E., Shapiro, K. L., \& Arnell, K. M. (1992). Temporary suppression of visual processing in an RSVP task: An attentional blink? Journal of Experimental Psychology: Human Perception and Performance, 18(3), 849-860.

Ricker, T. J., \& Cowan, N. (2014). Differences between presentation methods in working memory procedures: A matter of working memory consolidation. Journal of Experimental Psychology: Learning, Memory, and Cognition, 40(2), 417-428.

Ricker, T. J., Nieuwenstein, M. R., Bayliss, D. M., \& Barrouillet, P. (2018). Working memory consolidation: insights from studies on attention and working memory. Annals of the New York Academy of Sciences, 72, 11-89.

Ricker, T. J., \& Sandry, J. (2018). The relationship between masking and short-term consolidation during recall from visual working memory. Annals of the New York Academy of Sciences, 19, 411-450.

Schwarb, H., Nail, J., \& Schumacher, E. H. (2016). Working memory training improves visual short-term memory capacity. Psychological Research, 80(1), 128-148. 
https://doi.org/10.1007/s00426-015-0648-y

Shipstead, Z., Redick, T. S., \& Engle, R. W. (2012). Is working memory training effective? Psychological Bulletin, 138(4), 628-654.

Sørensen, T. A., \& Kyllingsbæk, S. (2012). Short-term storage capacity for visual objects depends on expertise. Acta Psychologica, 140(2), 158-163.

Sperling, G. (1960). The information available in brief visual presentations. Psychological Monographs: General and Applied, 74(11), 1-29.

Stevanovski, B., \& Jolicœur, P. (2007). Visual short-term memory: Central capacity limitations in short-term consolidation. Visual Cognition, 15(5), 532-563. https://doi.org/10.1080/13506280600871917

Stokes, M. G., Atherton, K., Patai, E. Z., \& Nobre, A. C. (2012). Long-term memory prepares neural activity for perception. Proceedings of the National Academy of Sciences of the United States of America, 109(6), E360-7.

Sun, H., Zimmer, H. D., \& Fu, X. (2011). The influence of expertise and of physical complexity on visual short-term memory consolidation. Quarterly Journal of Experimental Psychology, 64(4), 707-729. https://doi.org/10.1080/17470218.2010.511238

Turvey, M. T. (1973). On peripheral and central processes in vision: Inferences from an information-processing analysis of masking with patterned stimuli. Psychological Review, 80(1), 1-52. https://doi.org/10.1037/h0033872

Vidal, C., Content, A., \& Chetail, F. (2017). BACS: The Brussels Artificial Character Sets for studies in cognitive psychology and neuroscience. Behavior Research Methods, 49(6), 2093-2112. https://doi.org/10.3758/s13428-016-0844-8

Vogel, E. K., \& Luck, S. J. (2002). Delayed working memory consolidation during the attentional blink. Psychonomic Bulletin and Review, 9(4), 739-743. https://doi.org/10.3758/BF03196329

Vogel, E. K., \& Machizawa, M. G. (2004). Neural activity predicts individual differences in visual working memory capacity. Nature, 428(6984), 748-751.

Vogel, E. K., Woodman, G. F., \& Luck, S. J. (2006). The time course of consolidation in visual working memory. Journal of Experimental Psychology: Human Perception and Performance, 32(6), 1436-1451. https://doi.org/10.1037/0096-1523.32.6.1436

Wierda, S. M., Taatgen, N. A., van Rijn, H., \& Martens, S. (2013). Word Frequency and the Attentional Blink: The Effects of Target Difficulty on Retrieval and Consolidation Processes. PLOS ONE, 8(9). https://doi.org/10.1371/journal.pone.0073415

Woodman, G. F., \& Vogel, E. K. (2005). Memory Fractionating Consolidation and Maintenance Are Independent Processes. Psychological Science, 16(2), 106-113.

Woodman, G. F., \& Vogel, E. K. (2008). Selective storage and maintenance of an object's features in visual working memory. Psychonomic Bulletin and Review, 15(1), 223-229. https://doi.org/10.3758/PBR.15.1.223

Xie, W., Berry, A., Lustig, C., Deldin, P., \& Zhang, W. (2019). Poor sleep quality and compromised visual working memory capacity. Journal of the International Neuropsychological Society, 25(6), 583-594. https://doi.org/10.1017/S1355617719000183

Xie, W., Cappiello, M., Park, H. B., Deldin, P., Chan, R. C. K., \& Zhang, W. (2018). Schizotypy is associated with reduced mnemonic precision in visual working memory. Schizophrenia Research, 193, 91-97. https://doi.org/10.1016/j.schres.2017.07.046 
Xie, W., Li, H., Zou, Y., Sun, X., \& Shi, C. (2018). A suicidal mind tends to maintain less negative information in visual working memory. Psychiatry Research, 262, 549-557. https://doi.org/10.1016/j.psychres.2017.09.043

Xie, W., \& Zaghloul, K. A. (2021). Visual and Semantic Contributions to Visual Short-Term Memory. Trends in Cognitive Sciences, 25(4), 270-271. https://doi.org/10.1016/j.tics.2021.01.010

Xie, W., \& Zhang, W. (2017a). Dissociations of the number and precision of visual short-term memory representations in change detection. Memory and Cognition, 45(8), 1423-1437. https://doi.org/10.3758/s13421-017-0739-7

Xie, W., \& Zhang, W. (2017b). Familiarity increases the number of remembered Pokémon in visual short-term memory. Memory and Cognition, 45(4), 677-689. https://doi.org/10.3758/s13421-016-0679-7

Xie, W., \& Zhang, W. (2017c). Familiarity speeds up visual short-term memory consolidation. Journal of Experimental Psychology: Human Perception and Performance, 43(6), 12071221. https://doi.org/10.1037/xhp0000355

Xie, W., \& Zhang, W. (2018). Familiarity speeds up visual short-term memory consolidation: Electrophysiological evidence from contralateral delay activities. Journal of Cognitive Neuroscience, 30(1), 1-13. https://doi.org/10.1162/jocn_a_01188

Zhang, W., \& Luck, S. J. (2008). Discrete fixed-resolution representations in visual working memory. Nature, 453(7192), 233-235.

Zimmer, H. D., \& Fischer, B. (2020). Visual Working Memory of Chinese Characters and Expertise: The Expert's Memory Advantage Is Based on Long-Term Knowledge of Visual Word Forms. Frontiers in Psychology, 11, 516. https://doi.org/10.3389/fpsyg.2020.00516 